\title{
Are Antibiotics Prescribed to Inpatients According to Recommended Standard Guidelines in South Sudan? A Retrospective Cross-Sectional Study in Juba Teaching Hospital
}

\author{
Michael E Otim (iD) ${ }^{1,2}$ \\ Desmond Kabuya Demaya ${ }^{2}$ \\ Amina Al Marzouqi iD \\ Jean Mukasa ${ }^{3}$ \\ 'College of Health Sciences, University of \\ Sharjah, Sharjah, United Arab Emirates; \\ ${ }^{2}$ School of Graduate Studies and \\ Research, Nexus International University, \\ Kampala, Uganda; ${ }^{3}$ Fatima College of \\ Health Science, Institute of Technology, \\ Ajman, United Arab Emirates
}

\begin{abstract}
Purpose: Antibiotic resistance is spreading at an alarming rate globally, mainly because of antibiotics misuse. The World Health Organization developed guidelines for the rational use of antibiotics to prevent antibiotic misuse and reduce the potential development of antibiotic resistance. Although many countries adhere to these guidelines and have contextualized them to their needs, data on antibiotics use are limited in African countries, particularly in South Sudan. This study explored prescription patterns and use of antibiotics at Juba Teaching Hospital (JTH) to clarify the potential for antibiotic resistance in South Sudan.

Materials and Methods: We conducted a retrospective, cross-sectional study of archived patient data from 2016 to determine the prevalence of inappropriate antibiotics use at JTH. We used methodology developed in a previous study to assess the appropriate use of antibiotics. The study sample comprised 384 files. After reviewing and cleaning the files, 316 files were included in our analyses. This study was approved by the South Sudan Ministry of Health Ethics Review Board (approval number: MoH/ERB 51/2018) and all procedures were consistent with the Declaration of Helsinki.
\end{abstract}

Results: Antibiotics use was highest in the medical ward (75.4\%). Most antibiotics prescriptions were for infectious diseases $(23.7 \%)$, followed by ailments affecting the digestive system $(19.9 \%)$. Commonly prescribed antibiotics were ceftriaxone $(21.2 \%)$ and metronidazole $(20.0 \%)$. The mean number of antibiotics prescribed per patient encounter was 2.09 (95\% confidence interval: $1.98-2.19)$. Most files $(n=233,70.57 \%)$ demonstrated incorrect use of antibiotics with $78.8 \%(\mathrm{n}=249)$ of prescriptions being inappropriate (misuse).

Conclusion: This study revealed a high level of inappropriate antibiotics use at JTH despite the existence of local guidelines, which suggested there was an increased risk for antibiotic resistance. Therefore, it is necessary to introduce antibiotic stewardship activity, along with continuous national surveillance. Enforcement of guidelines to reduce irrational antibiotics use may reduce the risk for antibiotic resistance.

Keywords: antibiotics, low-income countries, clinical guidelines, policy implications, antimicrobial resistance, AMR, antibiotic use, antibiotic dispensing

\section{Introduction}

Antimicrobial resistance (AMR) is spreading at an alarming rate globally. This is mainly attributed to over-prescription of antibiotics to patients without due indication, and accounts for about 700,000 deaths per year. ${ }^{1}$ O'Neill ${ }^{2}$ estimated that by 2050, AMR will result in approximately 10 million lost lives lost and cost US\$100
Correspondence: Michael E Otim

College of Health Sciences, University of Sharjah, P.O. Box 27272, Sharjah, United Arab Emirates

Tel +97l 65057527

Email motim@sharjah.ac.ae 
trillion per year worldwide. Furthermore, Gould et $\mathrm{al}^{3}$ and Bartlett et $\mathrm{al}^{4}$ estimated that multidrug resistance will have caused nearly 444 million deaths by 2050 . Other contributors to AMR are the use of antimicrobials in the food and livestock industries, ${ }^{5}$ including the use of antibiotics in food production (animals and plants) to prevent, control, and treat disease and promote growth. ${ }^{6}$

However, recent evaluations of AMR-related costs had restricted scopes, and further prospective studies are needed to estimate the actual economic burden. In theory, microbes develop resistance to antibiotics through a natural selection process wherein some microbes succumb to an antibiotic whereas others do not. ${ }^{7}$ Misuse of antimicrobials often exacerbates this process. Generally, first- and second-line antibiotic treatment options limit resistance and availability of effective antibiotics. Patients with resistant infections are more likely to die, and those that survive may have extended hospital stays, delayed recuperation, and long-term disability. ${ }^{8}$ Achievements in reducing mortality and morbidity through early use of antibiotics based on practical guidelines have also jeopardized appropriate actions to control AMR. ${ }^{9}$

In developing countries, approximately $60 \%$ of medicines in public health facilities and $70 \%$ in private facilities are prescribed and dispensed inappropriately. ${ }^{10}$ Recent studies reported increased antibiotic abuse in developing countries where irrational antibiotics use has become common, with (often unnecessary) antibiotics prescribed for $44-97 \%$ of hospitalized patients. ${ }^{11}$ A study conducted in Bangladesh found that $38 \%$ of patients received antibiotics, of which $14 \%$ of prescriptions were irrational. ${ }^{12}$ Baktygul et al also reported inappropriate use of antibiotics (73.3\%) in a hospital in the Kyrgyzstan Republic, with significantly higher inappropriate antibiotics choice in gynecology (odds ratio 2.70, 95\% confidence interval [CI]: 1.02-7.69) compared with other wards. ${ }^{13}$

A lack of quality data and weak AMR surveillance systems mean that the magnitude of AMR in Africa is not fully understood. However, Essack et al noted that the high burden of communicable diseases in Africa engenders extensive use of antibiotics, which leads to AMR. ${ }^{14}$ For example, a study from Ghana reported high bacterial resistance to certain drugs, including chloramphenicol, tetracycline, ampicillin, and cotrimoxazole $(>70 \%) .{ }^{15}$ In Cameroon, Chem et al reported an antibiotics prescription rate of $36.71 \%$, with a mean of 1.14 antibiotic prescriptions per patient per year. ${ }^{16}$ Those authors concluded there was the misuse of antibiotics in primary care facilities and recommended that only physicians should be allowed to write prescriptions because they were adequately trained.

Another study from East Africa reported high levels of AMR to commonly used antibiotics (ampicillin and cotrimoxazole), with resistance rates of $50-100 \% .{ }^{17}$ However, no such study has been conducted in South Sudan to guide government policy on antibiotics use, and no data on antibiotic use are available. Despite the existence of protocols guiding the use of medicines in the healthcare system, there are unconfirmed reports of patients being prescribed antibiotics and other medicines without microbiological testing. In addition, many prescribers do not follow available guidelines or protocols in their day-today practice, resulting in incorrect prescribing and use of antibiotics. Therefore, it is necessary to assess the use of antibiotics in South Sudan to provide scientific evidence regarding the magnitude of this problem.

Antibiotics account for a substantial proportion of hospital drug expenditure, and their misuse and overuse generates unnecessary costs. Among reported issues associated with the inappropriate prescribing of antibiotics, knowledge deficits among prescribers and problematic or underequipped practice environments have been highlighted. ${ }^{18}$ Shafiq et al ${ }^{19}$ found that implementing treatment guidelines for commonly occurring infections in the tertiary care hospital setting increased the appropriate use of antibiotics. The World Health Organization (WHO) noted that many prescribers in developing countries have little access to proper information about diagnosis and drugs. ${ }^{20}$ For example, the South Sudan Standards for Treatment Guidelines (STG) are often unavailable in facilities, and health workers are often unsupported and unsupervised ${ }^{6 .}$ These findings supported the rationale for this study.

Healthcare costs for antibiotic-resistant infections are markedly higher than those for patients with non-resistant infections. ${ }^{21}$ In Africa, AMR is an acknowledged problem in the treatment of HIV and pathogens that cause malaria, Tuberculosis, typhoid, cholera, meningitis, gonorrhea, and dysentery. ${ }^{22}$ It is therefore crucial for African countries to develop AMR plans. Worldwide, few countries (4.3\%) have national AMR plans and only $14.9 \%$ have national infection prevention control policies, although $93.6 \%$ have essential medicines lists, and $91.5 \%$ have national medicines policies and treatment guidelines reflecting rational use. ${ }^{14}$ However, no countries have national surveillance systems that routinely generate representative, robust data on antimicrobial use and resistance. ${ }^{14}$ In response to calls 
for urgent action, the World Health Assembly adopted a Global Action Plan on AMR in May 2015. The Africa Centers for Disease Control and Prevention (CDC) also established the Anti-Microbial Resistance Surveillance Network (AMRSNET). ${ }^{22}$ The goals of AMRSNET for the subsequent 5 years were to improve surveillance of AMR organisms among humans and animals, delay AMR emergence, limit AMR transmission, and mitigate harm among patients infected with AMR organisms.

A previous study noted that measuring the level of rational antibiotic prescribing contributes to understanding the correct use of antibiotics in a country and preventing the development of $\mathrm{AMR}^{10}$ To address the problem of $\mathrm{AMR}$, the $\mathrm{WHO}^{20}$ advocates for the rational use of drugs based on the "Rule of Right." This rule focuses on providers ensuring their prescriptions reflect proper treatment, correct doses, and a suitable duration. It also assumes patients adhere to the treatment regimens prescribed, including completing the dose. This means that physicians must give "the right drug to the right patient at the right time in the correct dose." However, this rule is often ignored by clinicians in Africa, leading to an increased risk for AMR. Most healthcare services in South Sudan are publicly funded and provided, and antibiotics use is limited to prescription and regulated overthe-counter access. In this context, it is urgent to address the problem of AMR because new antibiotic-resistant mechanisms are emerging that threaten our ability to treat common bacterial diseases and result in prolonged illness, thereby increased treatment costs, disability, and death. This study will provide guidance for policymakers, health professionals, and prescribers regarding implementing strategies to help contain antibiotics resistance in South Sudan.

\section{Materials and Methods}

This study explored the magnitude of over- and underprescription/use of antibiotics in Juba Teaching Hospital (JTH), which is located in South Sudan, to estimate the risk for AMR. We defined appropriate antibiotics use as use that was consistent with WHO rational use or national treatment guidelines. ${ }^{20}$ We used the model developed by Gyssens et $\mathrm{al}^{26}$ to evaluate whether the prescription of antibiotics was compliant with the South Sudan STG. We referred to the STROBE guidelines in preparing this report. $^{23}$

\section{Study Design}

This study used a retrospective cross-sectional design. We used data from archived files for patients admitted to the medical and surgical wards at JTH between January and December 2016. A non-probability purposive sampling process was used to select files for review.

\section{Study Setting}

This study was conducted at JTH, which is located in Juba, Juba County, Jubek State (Former Central Equatoria State). Juba is the capital city of the Republic of South Sudan. JTH is a public tertiary-care teaching institution that has an official capacity of 260 beds and covers various medical specialties. We focused on admissions to the medical and surgical wards, including those for children, because the pediatric ward was not functional at that time.

\section{Study Population}

This study focused on inpatients who had been prescribed antibiotics at JTH, irrespective of their condition. The hospital records indicated that in 2016, the total number of admissions was 40,251. We searched the files extracted from the hospital archives to identify files for patients admitted to the medical and surgical wards between January and December 2016.

\section{Sample Size and Description}

We accessed files for children (aged 0-18 years) and adults (aged $>18$ years) admitted to the JTH medical and surgical wards. The required sample size was 384 files (at a 95\% confidence level), which was estimated using the United States CDC calculator (StatCalc Epi Info ${ }^{\mathrm{TM}}$ ). ${ }^{24}$ We excluded 68 files because of insufficient information, which left 316 files for review (113 males and 203 females).

\section{Ethical Approval}

We could not obtain informed consent from patients as this was a retrospective study. However, this study followed the procedures set out in the Declaration of Helsinki ${ }^{25}$ and received approval from the Ministry of Health Ethics Review Board (approval number MOH/ERB 51/2018). We used data from de-identified files. First, the researchers formally contacted the study hospital by letter to obtain consent to use archived files for this study. This letter included the detailed study protocol and a statement outlining the study's purpose, and potential benefits to the hospital and country. The hospital consented to participate in this study. 


\section{Data Collection}

To avoid possible bias, files were drawn from the archived medical and surgical ward files using simple nonprobability sampling proportional to the ward size. The files required for review were then selected from the ward files using a simple random sampling method. We collected secondary data from the selected files over 2 months using a standard data collection form adopted from Baktygul et al. ${ }^{13}$ Compliance behavior was assumed to be under the supervision of ward nurses as they attended to the patients. For example, when distributing oral medication (antibiotics), nurses provided patients with water to swallow the medication, thereby ensuring compliance.

The data collection team had medical backgrounds and received 2 days of training and orientation using the data collection form before this study. The collected data were entered into a computer database, cleaned, and then assigned codes. Demographic characteristics recorded included the patients' age, gender, and diagnosis. Variables included in the quality assessment of antibiotics use were antibiotics prescription, dose, route of administration, duration, and the correct choice of antibiotics. We evaluated the indications for antibiotic use based on adherence to the South Sudan guidelines.

\section{Data Analysis and Interpretation}

Clinical microbiology services provided data to measure resistance to antimicrobial agents. As we used archived data based on cumulative tabulated susceptibility testing results of isolates from individual patients, we evaluated antibiotics use with an algorithm developed by Gyssens et al. ${ }^{26}$ This model helped us assess the prevalence of appropriate antibiotic use in the reviewed files as an outcome. The variables assessed for the prescribed antibiotics were correctness/incorrectness of choice, duration of therapy, dosage, and route of administration. Correctness or appropriateness implied that the antibiotic choice, duration of therapy, dosage, and route of administration were all correct. Data for the prescribed antibiotics were compared with the STG and standard textbooks to evaluate correct antibiotic use. $^{27}$ The collected data were then edited, coded, tallied, cleaned, and scored.

We used descriptive statistics to address our study objective. STATA version 13.0 was used for the data analyses. ${ }^{28}$ Numbers were used to code data for categorical variables and frequencies and percentages were used to present quantitative data, with the main results shown in tables. We evaluated the appropriateness of antimicrobial treatment using the algorithm developed by Gyssens et al. ${ }^{26}$ To understand the level of misuse, we examined patients' characteristics, type of antibiotics used, and patients' clinical conditions. We chose this algorithm because the method was validated in a previous study. ${ }^{29}$ In brief, this algorithm is judged as follows.

A. Appropriate decisions: all criteria of correct antimicrobial use were fulfilled.

B. Inappropriate indication: prescription of antimicrobials without the presence of infectious disease or for an infection that did not need antimicrobial treatment.

C. Inappropriate choice, including the inappropriate spectrum of the antimicrobial agent (too broad, too narrow, not practical), or inappropriate toxicity profile.

D. Inappropriate application, including inappropriate dosage, timing, route of administration, and duration of therapy.

E. Divergence from guidelines.

F. Missing or insufficient data to judge the appropriateness of antimicrobial use.

\section{Results}

The primary aim of this study was to clarify the magnitude of antibiotics misuse at JTH and estimate the risk for AMR based on national treatment guidelines. We described the characteristics of the study population, antibiotics used, and medical conditions treated. We assessed the quality of antibiotics use based on the dose, route of administration, duration of treatment, and choice of antibiotic. We also evaluated whether the antibiotics prescribed were consistent with the national STG.

\section{Patients' Demographic and Clinical Characteristics}

We reviewed the medical records for 384 patients to determine the use of antibiotics during their hospital stay. From these, we included the medical records for 316 patients that had complete information in our analyses. Table 1 shows patients' characteristics. About $64.2 \%$ of patients were female, and over half were aged $\geq 18$ years. The distribution of patients in the selected wards was non-homogeneous, with the medical wards having the highest number of admissions $(n=248$, $78.5 \%$ ). Both the medical and surgical wards had more female than male patients. 
Table I Patients' Demographic and Clinical Characteristics

\begin{tabular}{|l|l|l|}
\hline Variable & Frequency (n) & Percentage (\%) \\
\hline Gender & 113 & 35.76 \\
Male & 203 & 64.24 \\
Female & \multicolumn{2}{|l}{} \\
\hline Age group, years & 94 & 29.75 \\
\hline $0-5$ & 25 & 7.91 \\
$6-17$ & 197 & 62.34 \\
$\geq 18$ & \multicolumn{2}{|l}{} \\
\hline Ward & 248 & 78.48 \\
& 68 & 21.52 \\
\hline Medical & \multicolumn{2}{|l}{} \\
Surgical &
\end{tabular}

Table 2 Most Commonly Prescribed Antibiotics

\begin{tabular}{|l|l|l|}
\hline Antibiotic & Frequency (n) & Percentage (\%) \\
\hline Penicillin & 223 & 34 \\
Cephalosporin & 152 & 23 \\
Metronidazole & 132 & 20 \\
Aminoglycoside & 75 & 11 \\
Quinolone & 38 & 6 \\
Macrolide & 20 & 3 \\
Other (tetracycline, TMP- & 20 & 3 \\
SMX, chloramphenicol, and & & \\
nitrofurantoin) & & \\
Total & 660 & 100 \\
\hline
\end{tabular}

\section{Conditions for Which Antibiotics Were Prescribed}

In both wards, patients were administered antibiotic/antimicrobial regimens for infections in all organ systems. Many patients were diagnosed with infectious and parasitic diseases $(n=75,23.7 \%)$, including diseases of the digestive system $(n=63,19.9 \%)$ and respiratory system $(n=56,17.7 \%)$, all of which were treated with antibiotics and protozoal agents. Those without parasitic infection only received antibiotic treatment. Eight different antibiotic groups and 21 single antibiotics were prescribed (Table 2). Most prescribed antibiotics (34\%) were from the penicillin group, followed by the cephalosporin (23\%) and metronidazole groups (20\%). Only $11 \%$ of prescribed antibiotics were from the aminoglycoside group.

\section{Prevalence of Antibiotic Use by Ward}

The frequency of single antibiotics prescribed by ward type is shown in Table 3. There were 660 prescriptions
Table 3 Prevalence of Single Antibiotic Use at Juba Teaching Hospital

\begin{tabular}{|l|l|l|}
\hline Antibiotic (Single) & Frequency (n) & Percentage (\%) \\
\hline Ceftriaxone & 140 & 21 \\
Metronidazole & 132 & 20 \\
Amoxicillin & 76 & 12 \\
Gentamicin & 74 & 11 \\
Benzylpenicillin & 71 & 11 \\
Others & 167 & 25 \\
Total & 660 & 100 \\
\hline
\end{tabular}

for single antibiotics across the studied wards, with ceftriaxone being the most commonly prescribed (21\%), followed by metronidazole and amoxicillin $(20 \%$ and $12 \%$, respectively). We found that 248 patients in the medical ward consumed $75 \%(n=497)$ of the antibiotics, and six patients in the surgical ward accounted for $25 \%(n=163)$ of the antibiotics used.

\section{Number of Antibiotics Prescribed per Patient During Their Admission Period} During their admission period, $40.2 \%$ of patients received two antibiotics, $30.1 \%$ received one antibiotic, and $20.6 \%$ received three antibiotics per prescription (Table 4). The average number of antibiotics prescribed per patient encounter was 2.09 (95\% CI: 1.98-2.19).

\section{Quality Assessment of Antibiotic Therapy}

We assessed whether the choice of antibiotics prescribed for each patient was correct or incorrect. We found that $29.43 \%(n=93)$ of the reviewed files showed a correct choice of antibiotics for therapy. However, 70.57\% $(n=223)$ of the files demonstrated incorrect use. In terms of dose, most files ( $\mathrm{n}=281,88.9 \%$ ) showed the dose of the prescribed antibiotics was correct, but 35 (11.1\%) files showed incorrect doses. The most commonly used administration method was injection solution (45.2\%), followed by tablet (25.2\%) and capsule (17.3\%) (Table 5).

Table 4 Average Number of Antibiotic Prescriptions per Patient

\begin{tabular}{|l|c|c|c|}
\hline Variable & Mean & $\begin{array}{c}\text { Std. } \\
\text { Error }\end{array}$ & $\begin{array}{c}\text { 95\% Confidence } \\
\text { Interval }\end{array}$ \\
\hline Antibiotic & 2.094937 & 0.0533348 & $1.989999-2.199874$ \\
Medical ward & 2.012097 & 0.0600601 & $1.893927-2.130266$ \\
Surgical ward & 2.397059 & 0.1091505 & $2.182303-2.611815$ \\
\hline
\end{tabular}


Table 5 Modes of Antibiotic Delivery

\begin{tabular}{|l|l|l|}
\hline Dosage Form & Number (n) & Percentage (\%) \\
\hline Syrup/suspension & 62 & 12.00 \\
Capsule & 89 & 17.30 \\
Tablet & 130 & 25.20 \\
Injection solution & 233 & 45.20 \\
Other & 2 & 0.40 \\
Total & 516 & 100.00 \\
\hline
\end{tabular}

As shown in Table 6,74.1\% of patients received antibiotic therapy during an admission period of 0-7 days, $21.5 \%$ received treatment in an admission period of 8-14 days, and $4.4 \%$ received antibiotic treatment in an admission period $\geq 15$ days.

\section{Rationality of Antibiotic Therapy at JTH}

Our review of the single antibiotics used showed the majority of antibiotics were given intravenously $(51.5 \%)$, followed by oral $(47.6 \%)$ and intramuscular $(0.66 \%)$ administration. Parenteral antibiotics were also commonly used. Antibiotic therapy was correctly used (appropriate) in $21.2 \%(n=67)$ of the prescriptions, and incorrectly used (inappropriate) in $78.8 \% \quad(\mathrm{n}=249)$ of prescriptions. Antibiotics prescribed were evaluated by diagnosis to clarify if they were compliant with local treatment guidelines. However, only $29.11 \%(\mathrm{n}=92)$ of prescriptions complied with treatment according to these guidelines, and $70.89 \%$ $(\mathrm{n}=224)$ did not comply.

\section{Discussion}

The primary aim of this study was to explore the prevalence of antibiotic misuse in South Sudan, which exposes the population to an increased risk for AMR. The results indicated that there was over-prescription of antibiotics in South Sudan. However, the socioeconomic conditions of the region mean it remains unclear if this translated to overuse or underuse. There are also government regulatory issues related to the availability of over-the-counter

Table 6 Duration of Therapy for Patients Admitted to Juba Teaching Hospital

\begin{tabular}{|l|l|l|}
\hline Admission Period & Number (n) & Percentage (\%) \\
\hline $0-7$ days & 234 & 74.10 \\
$8-14$ days & 68 & 21.50 \\
$\geq 15$ days & 14 & 4.40 \\
Total & 316 & 100.00 \\
\hline
\end{tabular}

antibiotics in the study setting, and the level of awareness of AMR among healthcare personnel remains unclear.

Few studies focused on AMR have been conducted in Africa, and none in the South Sudan setting. To fill this knowledge gap, this study evaluated how antibiotics were used in a hospital in South Sudan to elucidate the contribution of this prescribing practice/pattern to development of AMR. In the African region in general, understanding of issues related to AMR and its magnitude is hampered by the limited surveillance of drug resistance (only in a few countries), which has resulted in incomplete and inadequate data on the true extent of the problem. ${ }^{30}$ However, AMR is an acknowledged issue ${ }^{22}$ and countries should develop AMR plans. Unfortunately, few countries have national AMR plans (4.3\%) or national infection prevention control policies (14.9\%). Rates of essential medicines lists and national medicines policies and treatment guidelines reflecting rational use are higher $(93.6 \%$ and $91.5 \%$, respectively), but no countries have national surveillance systems that routinely generate representative, robust data on antimicrobial use and resistance. ${ }^{14}$ However, some progress has been made such as the Global Action Plan on AMR adopted by the World Health Assembly in May $2015,{ }^{30}$ and establishment of AMRSNET by the Africa CDC. ${ }^{31}$

Despite considerable improvement in the availability and control of antibiotics in hospitals, rational antibiotic use remains a worldwide concern. Increasing rates of AMR mean that medical intervention becomes impossible and people die of common infections (eg, during surgery, chemotherapy, organ transplantation, and care for premature infants). This study demonstrated high antibiotics use at JTH; antibiotics were commonly being misused (78.8\%) and non-adherence to local guidelines was high $(70.9 \%)$. The proportion of reviewed files in which antibiotics were prescribed at JTH was $100 \%$, with an average of 2.1 antibiotics per patient prescription. This finding was consistent with those reported by Ampaire et al, where AMR was high in commonly-used antibiotics (ampicillin and cotrimoxazole), with resistance of $50 \%-100 \%{ }^{17}$

This study also found that infectious and parasitic diseases were the most prevalent conditions for which antibiotics were prescribed, and there was a high rate of intravenous antibiotics administration (51.5\%). Our finding that the prescribed antibiotics were inappropriate in $78.8 \%$ of cases was consistent with studies from Ethiopia $(80.6 \%)^{32,33}$ and the Kyrgyz Republic (73.3\%). ${ }^{13}$ The most commonly used antibiotic group in our study was penicillin (33.8\%). We found that several inpatient records documented three $(20.6 \%)$ or four $(8.54 \%)$ antibiotics prescribed in a single encounter, 
with an average of 2.09 (CI: 1.98-2.19) antibiotics per prescription. This finding was comparable with studies from East Africa ${ }^{32-34}$ and Ghana, ${ }^{15}$ where patients received two antibiotics per encounter on average. In contrast, studies conducted in Saudi Arabia $^{35}$ and Cameroon ${ }^{16}$ reported a single antibiotic per prescription. The difference between those studies and this study may reflect limited time to diagnose the patient and select appropriate treatment. In addition, there may be differences in settings between our study (a tertiary hospital) and other studies.

Doctors may overprescribe antibiotics because of a lack of knowledge about rational use or to prevent potential infections. The 2.09 antibiotics prescribed per encounter in this study highlight the need for improved knowledge about polypharmacy among healthcare professionals, especially given the WHO recommended average of 1.6-1.8 antibiotics per encounter. The most common clinical indications for antibiotic use in this study were infectious and parasitic diseases (23.7\%). However, this was inconsistent with studies from Egypt $^{36}$ and Jordan, ${ }^{37}$ where the most prevalent conditions included respiratory tract (39.2\%) and urinary tract (53.75\%) infections. A study from India revealed that $69.4 \%$ of patients received antibiotics for acute respiratory infection and diarrhea of viral origin. ${ }^{19}$ These findings suggested that the burden of infectious and parasitic diseases in South Sudan contributed to the overuse of antibiotics. The increased burden of infections in developing countries may broadly explain the misuse of antibiotics in healthcare settings associated with AMR.

In this study, the majority of antibiotics (51.5\%) were given intravenously, followed by oral (47.6\%) administration, with overall injectable antibiotics constituting $45.2 \%$. Intravenous administration, including injectable antibiotics, exceeded the optimal level of $\geq 10 \%$ recommended by the WHO. ${ }^{14}$ However, the proportion of intravenous administration in our study was lower than that in a similar study in Pakistan, where injections accounted for $75 \%$ of antibiotics administration. ${ }^{37,38}$ However, the presence of pediatric patients in our study population might have contributed to the high use of intravenous antibiotics. Shifting the route of administration from an intravenous to an oral route saves costs, shortens the length of hospital stay, and decreases adverse reactions from intravenous use. ${ }^{33} \mathrm{We}$ found $74.1 \%$ of patients received antibiotic therapy during a $0-7$-day admission period, whereas $21.5 \%$ of patients received antibiotic treatment over 8-14 days and 4.4\% received treatment for 2 weeks. The cost of healthcare for patients with resistant infections is high because of a longer duration of illness, additional tests, and the use of more expensive drugs.

\section{Limitations of This Study}

This study had several limitations that should be noted. Despite inappropriate use of antibiotics and non-adherence to national treatment guidelines were high, we only included files for patients admitted to two wards in a tertiary-level hospital. This means our findings may not provide a comprehensive picture of antibiotics prescribing in South Sudan. In addition, the sample in this study may not be representative of the whole South Sudan population, which limits the generalizability of the results. Furthermore, we were not able to perform crosstabulation as part of our analyses. Finally, the data collectors involved in this study might not have been sufficiently knowledgeable about the data they were recording, despite having medical backgrounds and receiving training before data collection.

\section{Conclusions}

This study explored antibiotic prescribing practices in a hospital in South Sudan. Most antibiotics in the study hospital are prescribed for the treatment of infectious and parasitic diseases. However, we found antibiotics use in JTH is highly inappropriate, with prescribing patterns failing to adhere to national guidelines. The proportion of antibiotics prescribed is $100 \%$, with an average of 2.1 antibiotics per patient prescription. The study area also has a high rate of intravenous antibiotics use. Our findings suggest it is necessary to introduce antibiotic stewardship activity in the study area, along with continuous national surveillance. Enforcement of guidelines to reduce irrational antibiotics use may be helpful to reduce the risk for antibiotic resistance.

\section{Data Sharing Statement}

The datasets generated and/or analyzed during the present study (which include individual treatment files) are not publicly available because of ethical restrictions but may be available from the corresponding author on reasonable request.

\section{Ethics Approval and Consent to Participate}

This study was approved by the Ministry of Health Ethics Review Board and given approval number MOH/ERB 51/ 2018. The study hospital was formally contacted (invitation letter sent) and consent sought for their participation. 
The invitation letter included the detailed study protocol and a statement outlining the study purpose, potential benefits and an estimated time for completion.

\section{Consent for Publication}

All authors consented to the publication of the study.

\section{Author Contributions}

All authors made a significant contribution to the work reported, whether that is in the conception, study design, execution, acquisition of data, analysis and interpretation, or in all these areas; took part in drafting, revising or critically reviewing the article; gave final approval of the version to be published; have agreed on the journal to which the article has been submitted; and agree to be accountable for all aspects of the work.

\section{Funding}

None to declare.

\section{Disclosure}

The authors report no conflicts of interest in this work.

\section{References}

1. Interagency Coordination Group (IACG) on Antimicrobial Resistance. No time to wait: securing the future from drug-resistant infections. Report to the Secretary General of the United Nations; 2019. Available from: https://www.who.int/antimicrobial-resistance/interagencycoordi nation-group/IACG_final_report_EN.pdf?ua=1. Accessed October 8, 2021.

2. O'Neill J. Tackling drug-resistant infections globally: final report and recommendations; 2016. Available from: http://amr-review.org/sites/ default/files/160525_Final\%20paper_with\%20cover.pdf. Accessed December 10, 2018.

3. Gould IM, Bal AM. New antibiotic agents in the pipeline and how they can help overcome microbial resistance. Virulence. 2013;4 (2):185-191. doi:10.4161/viru.22507

4. Bartlett JG, Gilbert DN, Spellberg B. Seven ways to preserve the miracle of antibiotics. Clin Infect Dis. 2013;56(10):1445-1450. doi:10.1093/cid/cit070

5. Founou LL, Founou RC, Essack SY. Antibiotic resistance in the food chain: a developing country-perspective. Front Microbiol. 2016;7:1881. doi:10.3389/fmicb.2016.01881

6. World Health Organisation. Essential Drugs Monitor, Double Issue No. 028 \& 029. CH-1211 Geneva 27, Switzerland: World Health Organization; 2000

7. Zaman SB, Hussain MA, Nye R, Mehta V, Mamun KT, Hossain N. A review on antibiotic resistance: alarm bells are ringing. Cureus. 2017;9(6):e1403. doi:10.7759/cureus.1403

8. Centers for Disease Control and Prevention (CDC). Office of infectious disease, antibiotic resistance threats in the United States; 2013. Available from: http://www.cdc.gov/drugresistance/threat-report-2013. Accessed September 19, 2019.

9. Tadesse BT, Ashley EA, Ongarello S, et al. Antimicrobial resistance in Africa: a systematic review. BMC Infect Dis. 2017;17:616. doi:10.1186/s12879-017-2713-1
10. Irunde $\mathrm{H}$, Minzi $\mathrm{O}$, Moshiro C. Assessment of rational medicines prescribing in health-care facilities in four regions of Tanzania. $J$ Pharm Pract Community Med. 2017;3(4):225-231.

11. Abdullah R. Antibiotic abuse in developing countries. Pharmaceut Reg Affairs. 2012;1:e106. doi:10.4172/2167-7689.1000e106

12. Begum T, Khan MI, Kawser S, et al. An audit of rational use of antibiotics in a tertiary hospital of Bangladesh. Delta Med Coll J. 2014;2(2):64-67. doi:10.3329/dmcj.v2i2.20526

13. Baktygul K, Marat B, Ashirali Z, Harun-or-Rashid MD, Sakamoto J. An assessment of antibiotics prescribed at the secondary health-care level in the Kyrgyz Republic. Nagoya J Med Sci. 2011;73:157-168.

14. Essack SY, Desta AT, Abotsi RE, Agoba EE. Antimicrobial resistance in the WHO African region: current status and roadmap for action. J Public Health. 2016;39:8-13.

15. Asante KP, Boamah EA, Abdulai MA, et al. Knowledge of antibiotic resistance and antibiotic prescription practices among prescribers in the Brong Ahafo Region of Ghana; a cross-sectional study. BMC Health Serv Res. 2017;17(1):422. doi:10.1186/s12913-017-2365-2

16. Chem ED, Anong DN, Akoachere J-FKT, Godman B. Prescribing patterns and associated factors of antibiotic prescription in primary health care facilities of Kumbo East and Kumbo West Health Districts, North West Cameroon. PLoS One. 2018;13(3):e0193353. doi:10.1371/journal.pone. 0193353

17. Ampaire L, Muhindo A, Orikiriza P, Mwanga-Amumpaire J, Bebell L, Boum Y. A review of antimicrobial resistance in East Africa. Afr J Lab Med. 2016;5(1):a432. doi:10.4102/ajlm.v5i1.432

18. Saha SK, Hawes L, Mazza D. Improving antibiotic prescribing by general practitioners: a protocol for a systematic review of interventions involving pharmacists. BMJ Open. 2018;8(4):e020583. doi:10.1136/bmjopen-2017-020583

19. Shafiq N, Praveen Kumar M, Gautam V, et al. Antibiotic stewardship in a tertiary care hospital of a developing country: establishment of a system and its application in a unit-GASP initiative. Infection. 2016;44(5):651-659. doi:10.1007/s15010-016-0913-z

20. World Health Organization. The Rational Use of Drugs: Report of the Conference of Experts Nairobi, 25-29 November 1985. Geneva; 1987.

21. World Health Organisation. Fact sheet. antibiotic-resistance; 2020. Available from: https://www.who.int/news-room/fact-sheets/detail/ antibiotic-resistance. Accessed October 8, 2021.

22. CDC (Centres for Disease Control) Africa. Framework for antimicrobial resistance, 2018-2023 AU; 2019. Available from: https//www.Africa CDC_AMR_Framework_Eng.pdf. Accessed July 12, 2019.

23. von Elm E, Altman DG, Egger M, Pocock SJ, Gøtzsche PC, Vandenbroucke JP. The Strengthening the Reporting of Observational Studies in Epidemiology (STROBE) Statement: guidelines for reporting observational studies. Prev Med. 2007;45 (4):247-251. PMID: 17950122. doi:10.1016/j.ypmed.2007.08.012

24. CDC SatCalc Epi Info $^{\text {TM }}$. Division of Health Informatics \& Surveillance (DHIS), Center for Surveillance, Epidemiology \& Laboratory Services (CSELS); 2019. Available from: https://www. cdc.gov/epiinfo/user-guide/statcalc/statcalcandopenepi.html.

Accessed October 8, 2021.

25. The World Medical Association. WMA declaration of Helsinki - ethical principles for medical research involving human subjects; 2021. Available from: https://www.wma.net/policies-post/wma-declarationof-helsinki-ethical-principles-for-medical-research-involving-humansubjects/. Accessed October 8, 2021.

26. Gyssens IC, van den Broek PJ, Kullberg BJ, Hekster Y, van der Meer JW. Optimizing antimicrobial therapy: a method for antimicrobial drug use evaluation. J Antimicrob Chemother. 1992;30:724-727. doi: $10.1093 / \mathrm{jac} / 30.5 .724$

27. MOH (Ministry of Health), Government of Southern Sudan. Prevention and treatment guidelines for primary health care centres and hospitals; 2006. Available from: http://www.southsudanmedicaljournal.com/assets/ files/misc/SS_Treatment_Guidelines07.pdf. Accessed October 8, 2021. 
28. STATA (Version 13). StataCorp LP; 2021. Available from: https:// stata-13.software.informer.com/. Accessed October 8, 2021.

29. Cusini A, Rampini SK, Bansal V, et al. Different patterns of inappropriate antimicrobial use in surgical and medical units at a tertiary care hospital in Switzerland: a prevalence survey. PLoS One. 2010;5 (11):e14011. doi:10.1371/journal.pone.0014011

30. World Health Organization. Global action plan on antimicrobial resistance; 2015. Available from: http://apps.who.int/iris/bitstream/ 10665/193736/1/9789241509763_eng.pdf. Accessed October 8, 2021.

31. African Union. Africa CDC framework for antimicrobial resistance. Available from: https://africacdc.org/download/africa-cdc-framework -for-antimicrobial-resistance/. Accessed October 8, 2021.

32. Abrha S, Assefa R, Molla F, et al. Antibiotic utilization and their cost in Ayder Referral Hospital, Mekelle, Ethiopia. Glob J Med Res. 2015;15(1):Version 1.0.

33. Getachew E, Aragaw S, Adissie W, Agalu A. Antibiotic prescribing pattern in a referral hospital in Ethiopia. Glob J Pharm Pharmacol. 2013;7(38):2657-2661.
34. Ndihokubwayo JB, Yahaya AA, Desta AT, et al. Antimicrobial resistance in the African region: issues, challenges and actions proposed. Key determinants for health in the African Region. Afr Health Monit. 2013;16:27-30.

35. Alharafsheh A, Alsheikh M, Ali S, et al. A retrospective cross-sectional study of antibiotics prescribing patterns in admitted patients at a tertiary care setting in the KSA. Int $J$ Health Sci. 2018;12(4):67.

36. Talaat M, Tamer Saied S, Amr Kandeel A, et al. A point prevalence survey of antibiotic use in 18 hospitals in Egypt. Antibiotics. 2014;3 (3):450-460. doi:10.3390/antibiotics3030450

37. Qasim S, Muqeet W. Evaluation of antibiotics use based on various parameters used for selection of antibiotics in Nishter Hospital Multan, Pakistan. J Appl Pharm. 2014;6(2):171-183.

38. Palikhe N. Prescribing pattern of antibiotics in paediatric hospital of Kathmandu Valley. Kathmandu Univ Med J. 2004;2(1):6.
Journal of Multidisciplinary Healthcare

\section{Publish your work in this journal}

The Journal of Multidisciplinary Healthcare is an international, peerreviewed open-access journal that aims to represent and publish research in healthcare areas delivered by practitioners of different disciplines. This includes studies and reviews conducted by multidisciplinary teams as well as research which evaluates the results or conduct of such teams or healthcare processes in general. The journal

\section{Dovepress}

covers a very wide range of areas and welcomes submissions from practitioners at all levels, from all over the world. The manuscript management system is completely online and includes a very quick and fair peer-review system. Visit http://www.dovepress.com/testimonials. php to read real quotes from published authors. 